\title{
Breastfeeding and birth weight are important predictors for childhood overweight and obesity: Results for 1885 6-7-year-old school-going children of the Childhood Growth Surveillance Initiative (COSI) in the Republic of Ireland
}

\author{
M. M. Heinen ${ }^{1}$, N. Eldin ${ }^{2}$, U. O’Dywer ${ }^{3}$, C. Hayes ${ }^{4}$, P. Heavey ${ }^{5}$, A. Lynam ${ }^{2}$, L. M. Mulhern ${ }^{1}$, \\ J. K. O’Brien ${ }^{6}$, M. C. O’Brien ${ }^{1}$, H. Scully ${ }^{1}$, L. E. Daly ${ }^{6}$, C. M. Murrin ${ }^{1}$ and C. C. Kelleher ${ }^{1}$ \\ ${ }^{1}$ National Nutrition Surveillance Centre, School of Public Health, Physiotherapy \& Population Science, University \\ College Dublin (UCD), Dublin, Ireland, ${ }^{2}$ Department of Health Promotion \& Improvement, Health Service Executive, \\ Ireland, ${ }^{3}$ Health Promotion Policy,Department of Health, Ireland, ${ }^{4}$ Public Health and Primary Care, Trinity College \\ Centre for Health Sciences, Dublin, Ireland, ${ }^{5}$ Athlone Institute of Technology, Athlone, Ireland and ${ }^{6}$ School of Public \\ Health, Physiotherapy \& Population Science, UCD, Dublin, Ireland
}

Obesity in children is an important health problem in Europe. It has been linked to adverse psychological, social and health consequences in childhood and later in life. Prevention is an important way to combat the childhood obesity epidemic. Even though literature seems clear on some factors that are related to childhood obesity, results regarding other factors including dietary factors are still inconsistent. $^{(1,2)}$ The WHO European Childhood Obesity Surveillance Initiative (COSI) was established in 2008 to measure systematically childhood obesity in the European region. Ireland is one of the countries that contribute to COSI. ${ }^{(3)}$ In the Irish study, data of 1885 (894 boys, 991 girls) Irish primary school children attending first class in 2010 or 2012, who underwent anthropometric assessment and whose parents filled out a family survey were included (response rate: $62 \cdot 1 \%$ of all children whose parents gave consent to fill out a questionnaire). The aim was to determine contributing factors, including several dietary factors, of childhood obesity.

Body mass index (BMI) and waist circumference (WC) were standardised by age and sex. For BMI, overweight (including obesity) was classified using the International Obesity Taskforce cut-off points. For WC, British 1990 data was used to compute z-scores and overweight was defined as a $\mathrm{z}$-score $\geq 1.33$ ( $91^{\text {st }}$ centile). Parents filled out a questionnaire on several factors including the child's dietary intake frequency of several food items, physical activity/inactivity pattern of the child, and family's socioeconomic characteristics. Backward stepwise elimination in the logistic regression was used to identify factors related to childhood obesity, using BMI in one model and WC in a second model as the outcome variable. These variables were dichotomised as normal versus overweight and obese combined. The STATA survey commands were used to adjust for sampling design.

Overweight and obesity combined using BMI as the outcome, were more likely in girls (OR 1.47, 95\%CI 1.07-2.03), children who were heavier at birth (kg: OR 1.56, 95\% CI 1.09-2.24), not breastfed (OR 1.66, 95\%CI 1.14-2.42), not living in detached houses/ bungalows (semi-detached house/ bungalow: OR 1.74, 95\%CI 1.30-2.32; apartment/shared/other: OR 3.53, 95\%CI 1.67-7.45). When using WC as the outcome, results were similar with overweight and obesity combined being more likely in girls, children who were heavier at birth and not living in detached houses/bungalows. Child's frequency of intake of dietary factors such as fruits, vegetables, soft drink (both sugary and diet), sweet snacks, salted snacks and fast food items as reported by the parent, were not associated with the outcomes. These findings might, however, be explained by other factors such as the possibility of self-report bias.

In the current study, early life factors and socio-economic status were associated with overweight and obesity, whereas dietary factors were not predictive in 6-7-year-old Irish children.

1. Weng S, Redsell S, Swift J et al. (2012) Arch Dis Child 97, 1019-26.

2. Pate R, O'Neill J, Liese A et al. (2013) Obes Rev 14, 645-58.

3. Wijnhoven T, Branca F (2008) WHO European Childhood Obesity Surveillance Initiate. Protocol, Version January 2008. Copenhagen: WHO Regional Office for Europe. 